Research Paper

\title{
Alveolar Type II Cells Escape Stress Failure Caused by Tonic Stretch through Transient Focal Adhesion Disassembly
}

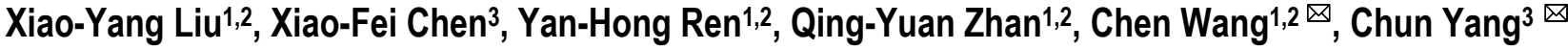 \\ 1. Beijing Chao-Yang Hospital, Capital Medical University, China \\ 2. Beijing Institute of Respiratory Medicine, China \\ 3. Institute of Biomechanics and Medical Engineering, School of Aerospace, Tsinghua University, China
}

$\triangle$ Corresponding author: Dr. Chen Wang, Beijing Chao-Yang Hospital, Capital Medical University, Beijing, 100020 (China). Dr. Chun Yang, IBME, School of Aerospace, Tsinghua University, Beijing 100084 (China). Tel. +86 1085132114 ; Fax +86 10 6506 0167; E-Mail: cyhbirm@mail.ccmu.edu.cn or yangchun@tsinghua.edu.cn

(c) Ivyspring International Publisher. This is an open-access article distributed under the terms of the Creative Commons License (http://creativecommons.org/ licenses/by-nc-nd/3.0/). Reproduction is permitted for personal, noncommercial use, provided that the article is in whole, unmodified, and properly cited.

Received: 2011.01.10; Accepted: 2011.05.07; Published: 2011.05.11

\begin{abstract}
Mechanical ventilation-induced excessive stretch of alveoli is reported to induce cellular stress failure and subsequent lung injury, and is therefore an injurious factor to the lung. Avoiding cellular stress failure is crucial to ventilator-induced lung injury (VILI) treatment. In the present study, primary rat alveolar type II (ATII) cells were isolated to evaluate their viability and the mechanism of their survival under tonic stretch. By the annexin $\mathrm{V} / \mathrm{PI}$ staining and flow cytometry assay, we demonstrated that tonic stretch-induced cell death is an immediate injury of mechanical stress. In addition, immunofluorescence and immunoblots assay showed that the cells experienced an expansion-contraction-reexpansion process, accompanied by partial focal adhesion (FA) disassembly during contraction. Manipulation of integrin adherent affinity by altering bivalent cation levels in the culture medium and applying an integrin neutralizing antibody showed that facilitated adhesion affinity promoted cell death under tonic stretch, while lower level of adhesion protected the cells from stretch-induced stress failure. Finally, a simplified numerical model was established to reveal that adequate disassembly of FAs reduced the forces transmitting throughout the cell. Taken together, these results indicate that ATII cells escape stress failure caused by tonic stretch via active cell morphological remodeling, during which cells transiently disassemble FAs to unload mechanical forces.
\end{abstract}

Key words: focal adhesion disassembly; cellular stress failure; cell morphologic remodeling; cell culture; tonic stretch

\section{Introduction}

Acute Respiratory Distress Syndrome (ARDS) treatment frequently leads to ventilator-induced lung injury (VILI), which is associated with $34-60 \%$ mortality [1]. Independent studies have documented that the mechanical ventilation used in the treatment of lung-injured patients may result in the aggravation of preexisting lung injury or de novo lung injury. VILI even occurs in normal lungs when high tidal volume artificial ventilation is performed [2].
The manifestations of VILI, such as edema, release of inflammatory factors, tissue rupture and fibrosis, can be attributed to defective cellular stress response induced by excessive strain [3]. Thus, avoiding defective cellular stress is crucial to VILI treatment. Tonic biaxial stretch induced defective cellular stress in the early stages of the stretch but not in later stages [4], suggesting that alveolar epithelial cells (AECs) could survive overstretch by certain 
mechanisms. Indeed, AECs recruit additional phospholipids via lipid trafficking to the plasma membrane to accommodate the required surface expansion[5] [6]. However, recent reports indicate some mechanisms beyond plasma membrane expansion. For instance, our previous high-resolution scanning electron microscopy (SEM) study showed that after a transient expansion A549 cells underwent a dramatic contraction-reexpansion with a large number of filaments scattered around the cell body [7]. Another independent study demonstrated that in AECs the fibers around the cell body formed a "tent-like" structure that is composed of actin filaments [8]. In addition, the reorganization of actin filaments in airway epithelial cells was closely associated with the survival of cells upon mechanical stimuli [9]. Taken together, these reports suggest a novel mechanism for the escape of AECs from cellular stress failure, but the details need further characterization.

Cell survival from tonic stretch, a biomechanical phenomenon, is proposed to be associated with intracellular stress bearing systems, namely actin bundles and FAs. Notably, FAs connect actin filaments to the extracellular matrix, where the mechanical stimuli act [10]. FA complexes consist of many signaling molecules (including src, Cas, vinculin and integrins) and are positioned at the upstream of the stress sensing pathway [11]. Thus, we hypothesize that cell adhesion, an upstream factor of actin filament reassembly, plays important roles in the ability of cells to survive overstretch.

In the present study, we aim to explore the mechanisms by which AECs escape stress failure. With our previously established in situ SEM analysis and FA staining, we demonstrated that FAs transient detachment is implicated in the early stage of the stretch induced-cells contraction characterized by tent like structure. The inhibition of $\beta 1$ integrin, a key adherent protein in FAs, showed that the adhesion property of the cells is closely associated with their survival from stress failure. Finally, a numerical model was built to predict the deformation and stress distribution in cells with different adhesion properties, and this model furthers our understanding of the relationship between cell adhesion and cellular stress failure under tonic stretch.

\section{Methods and Materials}

\section{Isolation and culture of rat ATII cells}

ATII cells were isolated according to established procedures $[4,12,13]$ approved by the Beijing experimental animal management center. Briefly, ATII cells were isolated from male Sprague Dawley rats (150-200g) by elastase digestion and differential adherence on IgG-coated dishes. The cells were seeded at a density of $1 \times 10^{6} / \mathrm{cm}^{2}$ on flexible silicone membranes mounted in custom-made wells [14] in culture medium (DMEM with 10\% FBS, $100 \mathrm{U} / \mathrm{ml}$ penicillin and $0.1 \mathrm{mg} / \mathrm{ml}$ streptomycin). ATII cells were identified using Nile Red (Sigma) staining of lamellar bodies [15], and $>95 \%$ of the cells were Nile Red positive on day 2. Experiments were performed on the $2^{\text {nd }}$ day after the isolation.

\section{Stretching pattern}

The stretching pattern was defined by the increase in surface area $(\triangle S A)$ of the calibrated silicone membrane. The cells were subjected to tonic stretch of $37 \% \Delta$ SA biaxial strain, which approximately corresponded to the strain experienced by AECs in vivo at $100 \%$ total lung capacity [16]. Unstretched cells served as controls.

\section{Cell viability assay}

Cell viability was assayed by the LIVE/DEAD viability/cytotoxicity kit (Molecular Probes, Eugene, OR, USA). Briefly, live cells were stained green with calcein AM, and dead ones were stained red by ethidium homodimer- III [7, 17]. The number of live/dead cells was counted in fluorescent images acquired randomly from four quadrants in each well under a fluorescence microscope $(\times 20$ objective, IX71, Olympus) with the Image-Pro Plus 6.0 software (Media Cybernetics, MD, USA). All experiments were from four cell isolations with duplicate measurements.

\section{Apoptosis assay}

The cells were harvested, stained with FITC-conjugated annexin $\mathrm{V}$ and propidium iodide (PI) using the Apoptosis Detection Kit (Biosea Biotechnology, Beijing, China), and analyzed by flow cytometry (BD Biosciences, San Jose, CA, USA). All experiments were from four cell isolations with duplicate measurements.

\section{Scanning electron microscopy}

The samples were prepared according to the protocol described previously [7]. In brief, a small glass sheet $\left(1 \mathrm{~cm}^{2}\right)$ was immediately glued to the back side of the silicone membrane before cell fixation to avoid membrane shrinkage. Samples were then dehydrated through graded ethanol series and critical-point dried (XD-1, Eiko, Japan) before the observation under SEM.

\section{Immunofluorescence}

The cells were subjected to immunostaining with 
mouse anti-vinculin (1:100; Sigma), TRITC-phalloidin (1:100; invitrogen), DAPI (1:5000; Sigma) and fluorescent-conjugated secondary Abs (Santa Cruz) [14]. The slides were mounted on a laser scanning confocal microscope (LSCM) (TCS-SP5, Leica, Wetzlar, Germany) for the observation.

\section{Western blot}

Cells were lysed and the lysate was resolved by 10\% SDS-PAGE, transferred onto PVDF membranes (Millipore, Bedford, MA, USA), and probed with anti-p-FAK (tyr397) (1:2000; Cell Signaling Technology, Danvers, MA, USA), total FAK (1:500), GAPDH (1:500) (Santa Cruz Biotechnology, CA, USA), followed by secondary Abs anti-mouse horseradish peroxidase (HRP) or anti-rabbit HRP (1:10000; Santa Cruz Biotechnology). Finally, the membranes were developed using a Kodak medical X-ray processor (Kodak, Rochester, NY, USA).

\section{Quantification of FA relative staining intensity}

Cells were fixed and stained with mouse anti-vinculin and FITC anti-mouse antibodies to label FAs, TRITC-phalloidin to determine the cell boundaries, and DAPI. Pictures were acquired on a confocal microscope at $63 \times$ objective using identical parameter for each type. Five random regions from each field were selected for analysis. 3-4 cells in the region were evaluated. ImageJ (National Institutes of Health, Bethesda, MD, USA) software was used to quantify the relative vinculin staining-FAs intensity. In brief, all images were converted into binary images and inverted binary images to isolate individual FA. An analysis was performed on thresholded images to selected classified objects of a size range of $0.1 \leq \mathrm{N} \leq 1$ E08 as FAs based on the anti-vinculin staining [18].

\section{Computational simulations}

Finite element analysis was performed using the commercial software ABAQUS (version 6.9; SIMULIA, Providence, RI, USA). ATII cell was modeled as a homogeneous viscoelastic standard linear solid model (SLS). In accordance with the previously established models $[19,20]$, the whole cell was simplified geometrically to an elliptical cylinder with a 40 $\mu \mathrm{m}$ long axis radius, $30 \mu \mathrm{m}$ minor axis radius, and 1.5 $\mu \mathrm{m}$ height [21]. Because the height was less than the other two axes, a plane stress model was used in the simulation. A zero-displacement boundary condition was imposed on some of the elements peripheral to the cell body to mimic FA sites.

\section{Statistical analysis}

Results were expressed as means \pm SD (ANOVA), followed by the Bonferroni test for post hoc analysis. Differences were accepted as significant for $\mathrm{P}<0.05$.

\section{Results}

\section{Tonic stretch induces time-independent cellular necrosis and morphological remodeling}

To investigate the mechanism of stretch-induced cell death, ATII cells were exposed to tonic biaxial stretch for $1 \mathrm{~min}, 5 \mathrm{~min}, 2 \mathrm{~h}$, and $4 \mathrm{~h}$, and the viability of the cells was evaluated using the Live/Dead Kit and fluorescence microscopy. Fig. 1A showed representative fluorescence images of live and dead cells at specific time points. Within the first $5 \mathrm{~min}$ after the stretch, cell death increased with time. However, cell death rate did not increase afterwards till $4 \mathrm{~h}$ (Fig. 1B). Mechanical stress has been shown to trigger the apoptotic pathway of cell death [13, 22]. In order to clarify whether the cells undergo necrosis or apoptosis, cells were analyzed by flow cytometry for annexin V/PI staining. As shown in Fig. 1C, there was no significant difference in the rate of early apoptosis or late apoptosis/necrosis between unstretched and stretched cells $(5 \mathrm{~min}, 2 \mathrm{~h}$ ). These findings indicate that stretch-induced cell death is an immediate injury of mechanical stress, and may result from extreme deformation of the cell membrane and cytoskeleton.

Next we observed the stretch induced-cell morphology changes under confocal microscope and high-resolution SEM. As shown in SEM images (Fig. 1D), ATII cells experienced an expansion-contraction-reexpansion response to tonic stretch $(2 \mathrm{~h})$. Particularly, the cells surface extended and tightly adhered to the substrate $(1 \mathrm{~min})$, and the cells dramatically contracted (5 min). Meanwhile, long filaments, called a "tent-like" structure, protruded from the cells bodies and attached to the substrate. $2 \mathrm{~h}$ after the stretch, the long filaments obviously decreased, and the cells spread again. Immunofluorescence images based on phalloidin staining of stretch induced-cell remodeling showed patterns similar to SEM observation (Fig. 1E). Furthermore, we performed latrunculin A to block actin filament assembly and the results shown in Fig. 2C. These results indicated that the tent-like structure is actin stress fiber around the cell body in the contraction stage. 
A

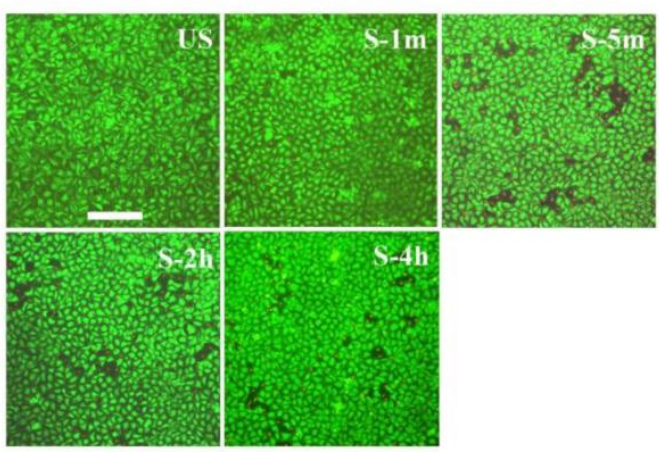

C

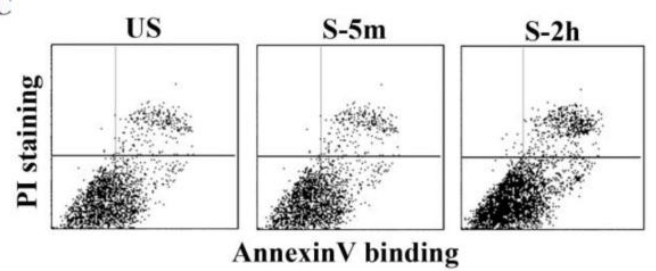

E

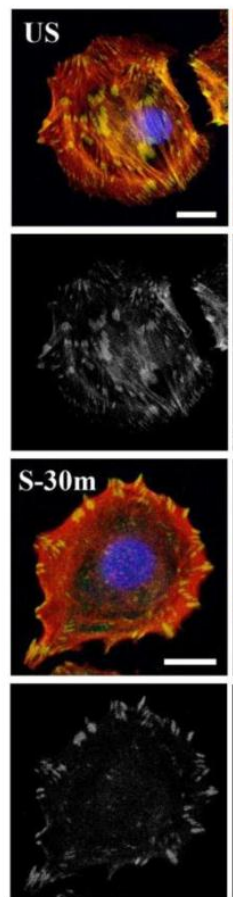

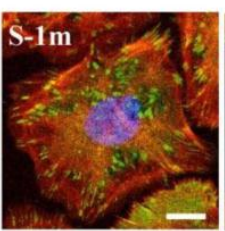
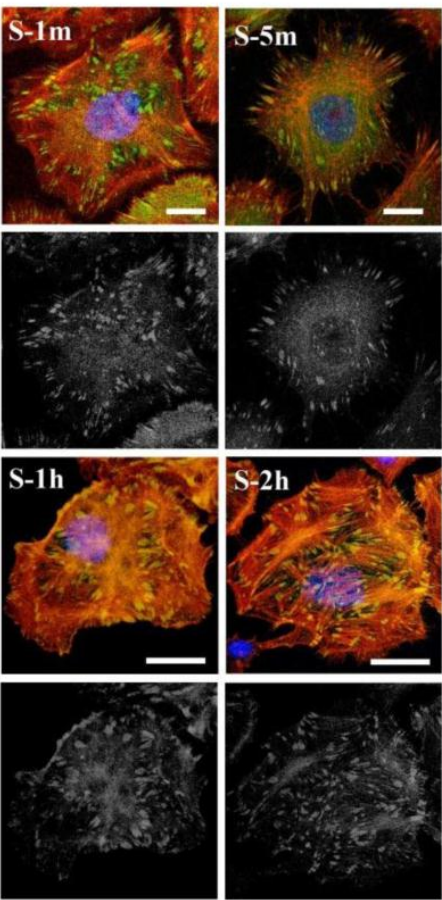

B
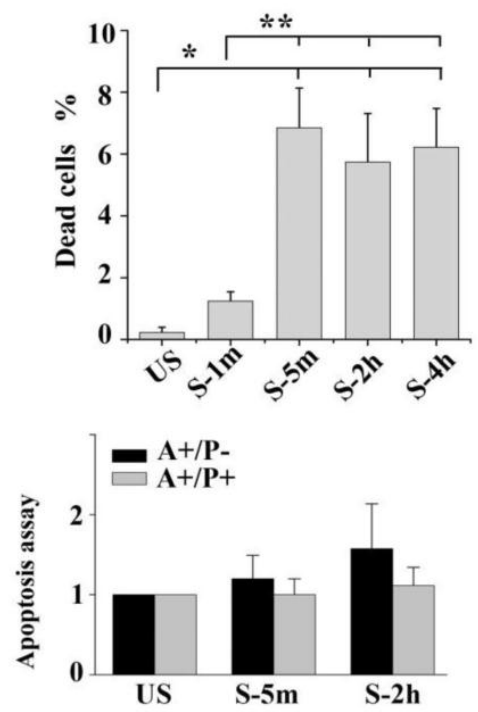

D

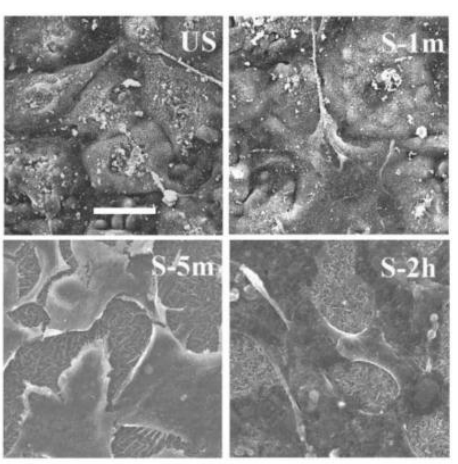

F

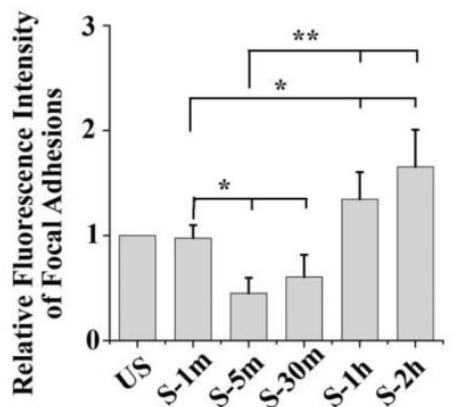

Figure I. (A) Tonic stretch-induced ATII cell death over time (I min, $5 \mathrm{~min}, 2 \mathrm{~h}$, and 4h). Cytoplasm of viable cells was stained with calcein-AM (green), and nuclei of nonviable cells were stained with ethidium homodimer-I (red). Bar $=200 \mu \mathrm{m}$. (B) Statistical analysis of stretch-induced cell death over time, the cell death rate did not increase after 5 min. Values were means $\pm S D(n=8)$. *P $<0.00$ I vs. unstretched cells (US); **P $<0.00$ I vs. S- Im. (C) Cells were stained with annexin V and PI, and analyzed by flow cytometry. AnnexinV-positive/PI-negative (lower right quadrant) represented the early apoptosis, annexinV-positive/PI-positive (upper right quadrant) represented the late apoptosis/necrosis. Apoptosis rate was not significantly different between US and stretch group (S-5m and S-2h). Values were means \pm SD ( $n=8)$. (D) Tonic stretch-induced cell morphological remodeling was detected by SEM. During the stretch, cells experienced expansion (I $\mathrm{min}$ ), contraction (5 min), and reexpansion $(2 \mathrm{~h})$. Bar $=20 \mu \mathrm{m}$. (E) Stretch induced cell morphological remodeling. Immunofluorescence of representative cells at the time indicated for actin (red), unclear (blue), and FA marker VIN (green). Bar $=10 \mu \mathrm{m}$. (F) Quantitative analysis of FAs fluorescence intensity during the stretch showed that cells experienced rapid FAs disassembly and sequential reassembly. $* P<0.0$ I vs. US; $* * P<0.0$ I vs. S-5m. Values were means $\pm S D$ ( $n>60$ ). 
A
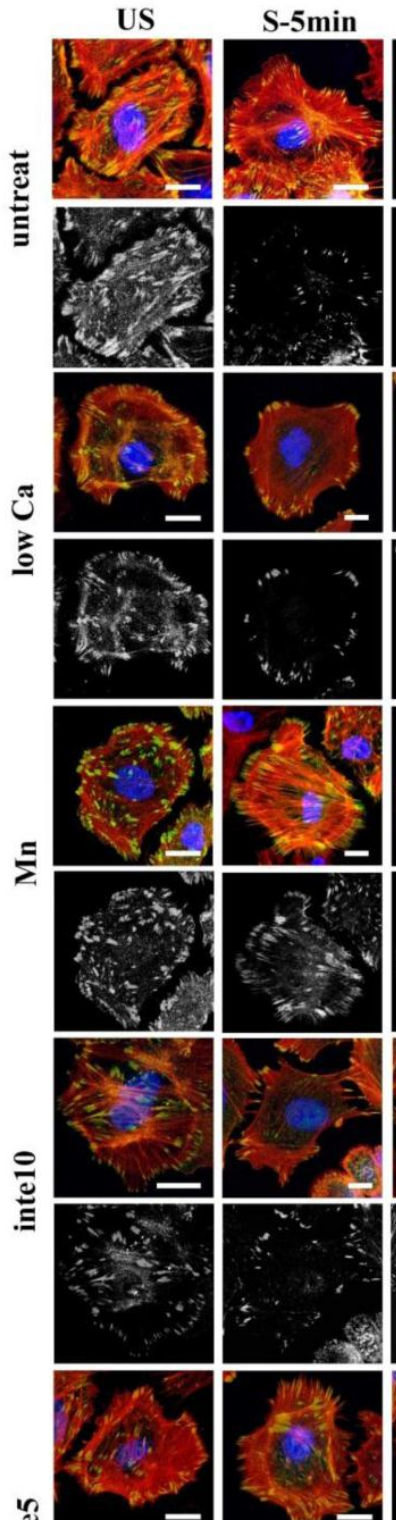

$\stackrel{\mathscr{E}}{\Xi}$
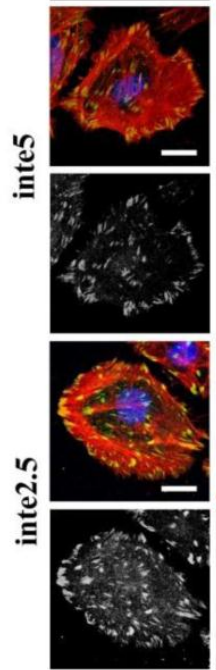

S-2h
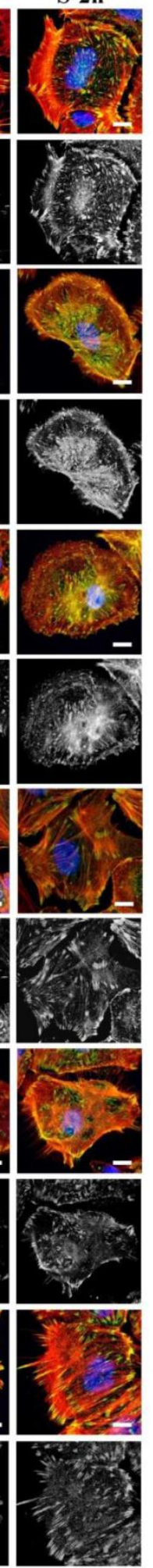

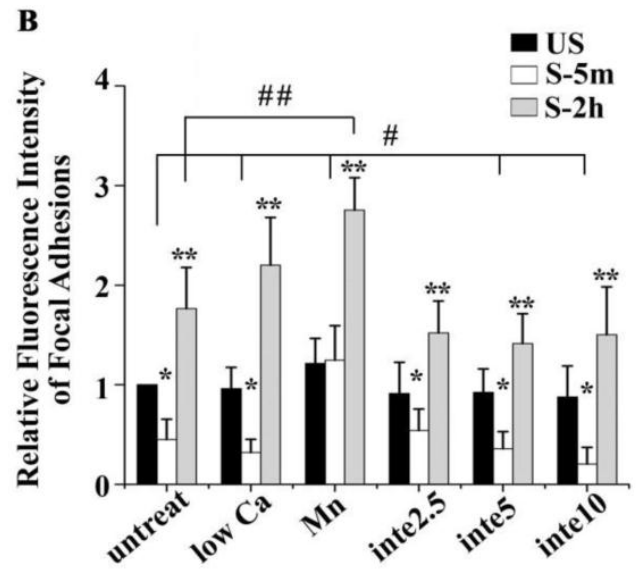

C

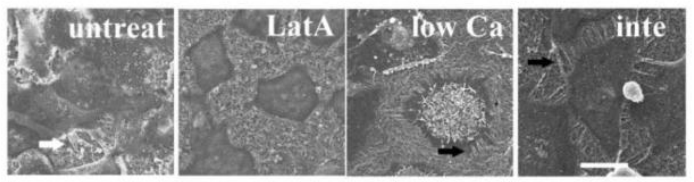

D
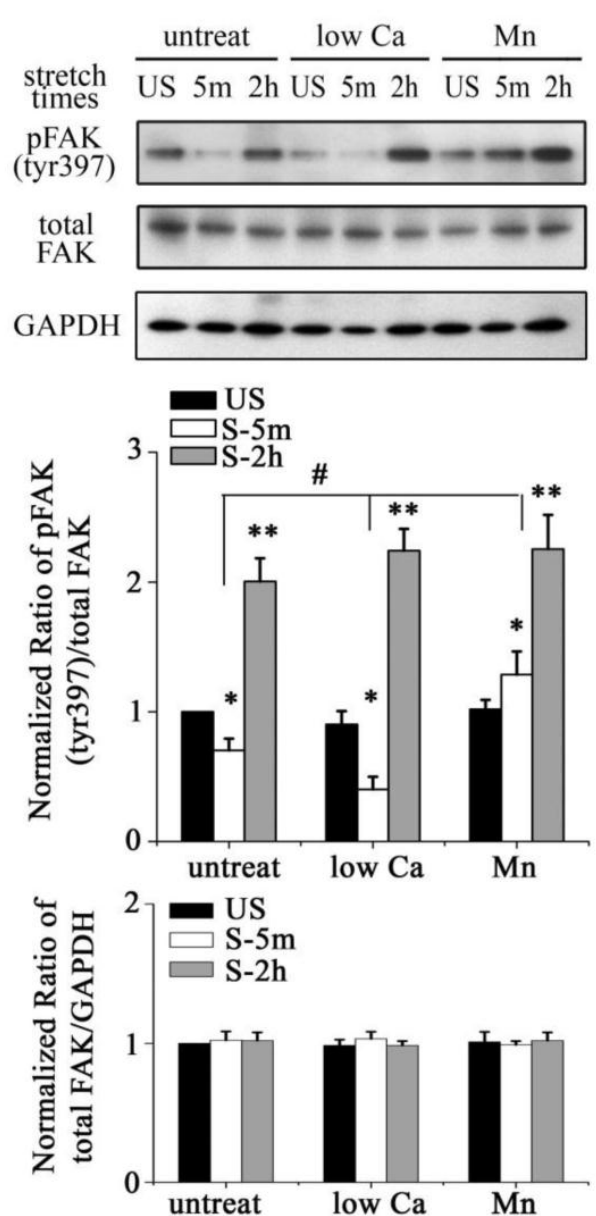

Figure 2. (A) Immunofluorescence of representative cells with different adhesion properties at the time indicated (time on $x$-axis) for actin (red), unclear (blue), and FA marker VIN (green). Low Ca indicated cells treated with medium with low $\mathrm{Ca}^{2+}$ concentration ( $1: 1$ mix of normal $\mathrm{Ca}^{2+} \mathrm{DMEM}$ and $\mathrm{Ca}^{2+}$-free DMEM). Mn indicated cells treated with $0.5 \mathrm{mM} \mathrm{Mn}^{2+}$ in addition to low $\mathrm{Ca}^{2+}$ medium. Inte2.5, inte5, inte 10 indicated cells pretreated with different concentrations of $\beta I$ integrin-neutralizing antibody at $2.5 \mu \mathrm{g} / \mathrm{ml}, 5 \mu \mathrm{g} / \mathrm{ml}$, and $10 \mu \mathrm{g} / \mathrm{ml}$ for $\mathrm{lh}$, respectively. Bar $=10 \mu \mathrm{m}$. (B) Quantitative analysis of relative fluorescence intensity of $F A$ in cells with different adhesion properties showed that low $\mathrm{Ca}^{2+}$ medium and $\beta$ I integrin-neutralizing 
antibody $(5 \mu \mathrm{g} / \mathrm{ml}, 10 \mu \mathrm{g} / \mathrm{ml})$ significantly increased FAs disassembly $(\# P<0.01$ vs. untreated cells). Mn treated cells significantly decreased FAs disassembly (\#\# $\mathrm{P}<0.0 \mathrm{I}$ vs. untreated cells). In addition, cells experienced detachment within 5 min after stretch $(* \mathrm{P}<0.0 \mathrm{I}$ vs. US) and then expansion within $2 \mathrm{~h}$ after stretch $(* * \mathrm{P}<0.0 \mathrm{l}$ vs. US). Values were means $\pm S D$ $(n>60)$. (C) SEM ultrastructural analysis of cells pretreated with latrunculin $A(L a t A)\left(2 \mu M\right.$ for I h), low $\mathrm{Ca}^{2+}$ medium (low $\mathrm{Ca}$ ), and the $\beta \mathrm{I}$ integrin antibody (inte) $(5 \mu \mathrm{g} / \mathrm{ml}$ for I h). In stretch induced cell contraction process long filaments appeared (marked by white arrow) around cell bodies in untreated cells, depolymerized in LatA cells, and enriched in low Ca or inte cells (marked by black arrow). Bar $=20 \mu \mathrm{m}$. (D) Western blots showing the levels of phosphorylated FAK and total FAK in different groups of cells. \#P $<0.05$ vs. untreated cells; $* P<0.05$ vs. US; $* * P<0.05$ vs. US. Values were means $\pm S D(n=4)$.

\section{Tonic stretch-induced cell contraction is due to focal ad-} hesion disassembly

We postulated that stretch induced tent-like structure is caused by the detachment of FAs. To test this, we performed immunofluorescence and immunoblot analysis of FAs protein to evaluate FAs dynamics. Immunofluorescence of vinculin-stained FAs (Fig. 1E) showed that upon the stretch FAs were distributed toward the cell periphery $(1 \mathrm{~min})$, then rapidly disassembled (5 $\mathrm{min}, 30 \mathrm{~min}$ ), and largely reassembled $(1 \mathrm{~h}, 2 \mathrm{~h})$. Quantitative analysis of FAs fluorescence intensity showed that cells underwent transient FAs disassembly and sequential reassembly during tonic stretch (Fig. 1F). FAK is a focal adhesion protein that undergoes self-phosphorylation when assembled into FAs. The level of FAK-tyr397 represents the amount of FA complex assembly [23, 24]. Under tonic stretch, the level of FAK-tyr397 rapidly decreased $(5 \mathrm{~min})$ and then largely restored $(2 \mathrm{~h})$ compared with unstretched cells (Fig. 2D). These results indicate that stretch induced cell contraction is due to rapid FAs disassembly. Notably, there is an interesting synchronization between the detachment/disassembly of FAs and cell viability. Within 5 min after stretch, cells underwent partial detachment, but cell death did not increase (Fig. 1B). We suspected that the FAs disassembly may be associated with the cell survival process from tonic stretch.

\section{FAs is crucial for cell survival during tonic stretch}

The binding of FAs to a substrate requires divalent cations. $\mathrm{Ca}^{2+}, \mathrm{Mg}^{2+}$ and especially $\mathrm{Mn}^{2+}$ can cause pronounced binding strength variation of FAs via influencing integrin conformation [25], and increasing the concentration of divalent cations in the culture medium can strengthen the cell attachment. To investigate the relationship of partial FA detachment and cell survival from overstretch, cells pretreated with medium containing different concentrations of divalent cations were exposed to tonic biaxial stretch. We evaluated the viability of cells treated with medium containing low $\left(0.9 \mathrm{mM} \mathrm{Ca}^{2+}, 1: 1 \mathrm{mix}\right.$ of normal $\mathrm{Ca}^{2+}$ DMEM and $\mathrm{Ca}^{2+}$-free DMEM) or normal concentrations of divalent cations. For the cells cultured in low $\mathrm{Ca}^{2+}$ medium, in which the integrin-ECM binding affinity was attenuated, cell death was reduced dramatically by 3 -fold at 5 min or $2 \mathrm{~h}$ after stretch, compared to the untreated stretched group at corresponding stretch time $(\mathrm{P}<0.001)$ (Fig. 3B), while relative FAs fluorescence intensity (Fig. 2B) and FAK-tyr397 protein level (Fig. 2D) decreased significantly. Notably, when the cell adhesion was weakened, intensive cell contraction during stretch was observed both by confocal microscopy and SEM (Fig. 2A and C). Moreover, contact area of low Ca treated cells at acute stretch time (1 min) was decreased compared with untreated or Mn treated cells $(\mathrm{P}<$ $0.05)$, which indicated that mechanical force transferred between substratum and cell is attenuated (Fig. S3).

To specify the role of adhesion in stretch-induced cell death, we inhibited FA assembly by using a neutralizing antibody against $\beta 1$ integrin. The pattern of FAs distribution in antibody treated cells was similar to low Ca treated cells, and was dose-dependent (Fig. 2A and B). The viability of the cells pretreated with $\beta 1$ integrin neutralizing antibody $(5 \mu \mathrm{g} / \mathrm{ml}$ for $1 \mathrm{~h})$ decreased dramatically to $29.6 \%$ (5 $\min )$ or $34.3 \%(2 \mathrm{~h})$ of untreated cells at the corresponding stretch time $(\mathrm{P}<0.001)$ (Fig. $3 \mathrm{~A}$ and $\mathrm{B})$. When the $\beta 1$ integrin neutralizing antibody concentration was increased to $10 \mu \mathrm{g} / \mathrm{ml}$, many cells immediately detached, and significant cell death was observed (Fig. 3A and B). According to apoptosis data (Fig. 3D), the significant cell death of integrin neutralizing antibody $(10 \mu \mathrm{g} / \mathrm{ml})$ pretreated was contributed to apoptosis mechanism. These results suggest that conservative FA detachment protects most cells from tonic stretch-induced stress failure, but excessive FA detachment causes cell death (apoptosis). 

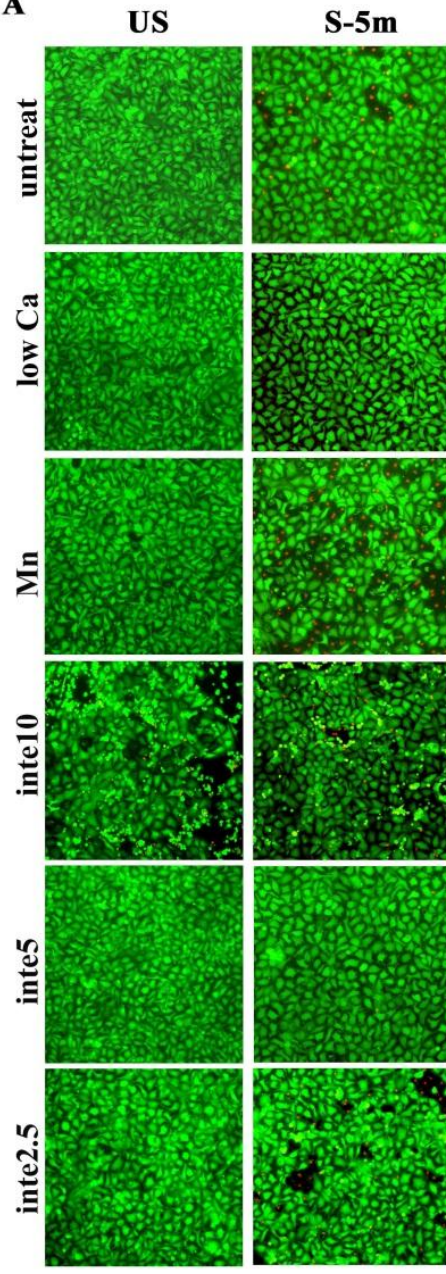

B
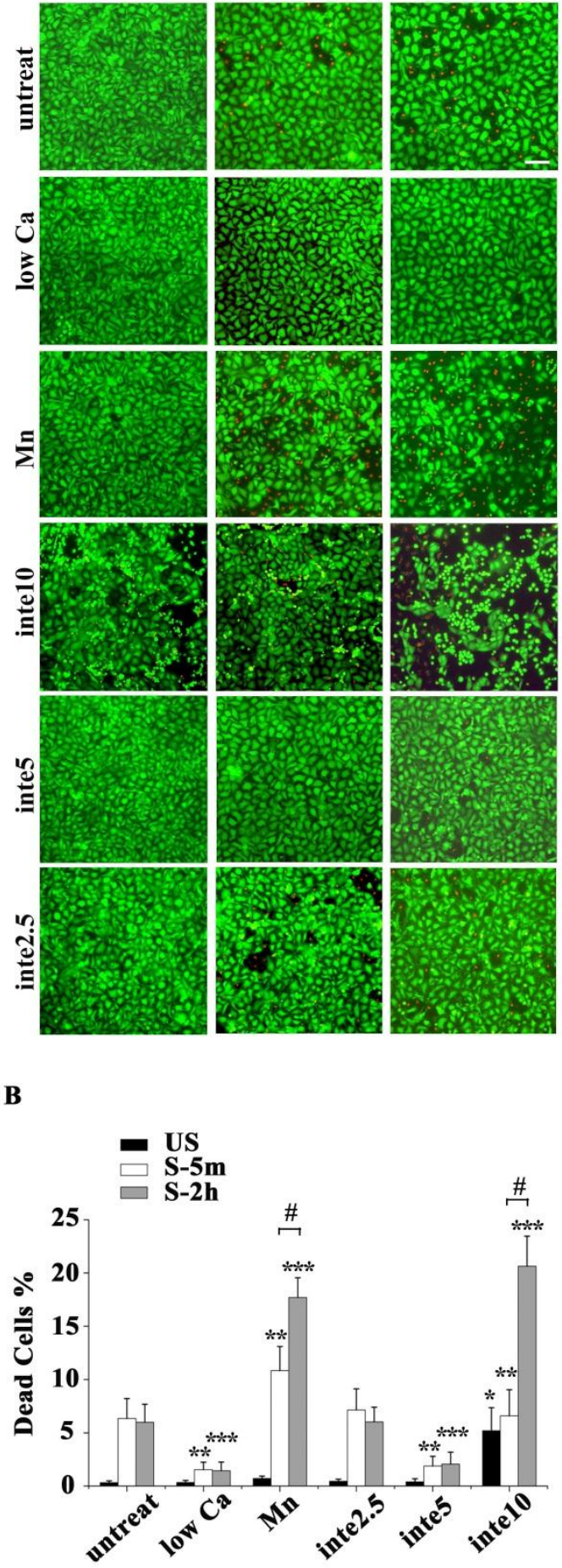

$\mathbf{C}$

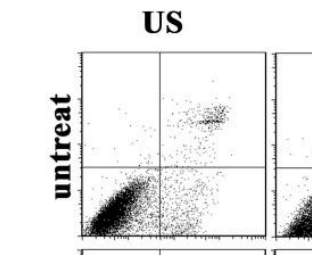

S-5m

S-2h

D

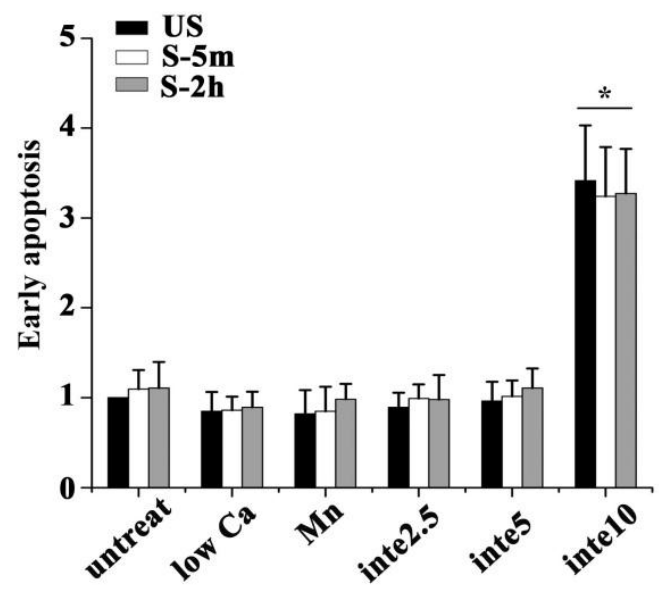

Figure 3. (A) The viability of cells with different adhesion properties at the time indicated (time on $\mathrm{x}$-axis). Bar $=100 \mu \mathrm{m}$. (B) Statistical analysis of cell death in different groups showed that partial cell detachment enhanced cell survival in tonic stretch, whereas strong cell adhesion increased cellular stress failure. $* P<0.001$ vs. untreated and unstretched cells; $* * P<$ 0.001 vs. untreated S-5m; *** $P<0.00$ I vs. untreated $S-2 h ; \# P<0.001$ vs. $S-5 m$. Values were means $\pm S D(n=8)$. (C) ATII cells with different adhesion properties were stained with annexin $\mathrm{V}$ and $\mathrm{PI}$, and analyzed by flow cytometry at the time indicated (time on $\mathrm{x}$-axis). (D) Statistical analysis of early apoptotic cells in different groups. $* P<0.05$ vs. untreated cells at the each time point. Values were means $\pm S D(n=8)$. 
We then wondered whether a high extent of cell adhesion would result in a decrease in cell viability after the stretch. As expected, enhanced cell adhesion induced by adding $\mathrm{Mn}^{2+}$, a specific agonist of integrin activation [26], into low- $\mathrm{Ca}^{2+}$ medium $\left(0.5 \mathrm{mM} \mathrm{Mn}{ }^{2+}\right.$ and $0.9 \mathrm{mM} \mathrm{Ca}^{2+}$ ) significantly decreased FAs disassembly during 5 min stretch (Fig. 2B and D) and increased the percentage of cell death with stretch time approximately 6-fold (5 $\mathrm{min})$ or 11-fold $(2 \mathrm{~h})$, compared to the low Ca group, respectively (Fig. 3A and B). Moreover, $\mathrm{Mn}$ treated cells did not undergo contraction after early stretch, as detected by confocal microscopy (Fig. 2A) and contact area assay (Fig. A3). These results suggest that FA dynamic is crucial to cell survival during tonic stretch.

\section{Variation in focal adhesion alters the forces transmitting throughout the cells}

Considering that tonic stretch-induced cell death is an immediate mechanical injury, we hypothesized that transient partial FA disassembly may alter the mechanical stress that propagates through the cells and thus protect the cells from overstretch-induced stress failure.

We introduced a viscoelastic plane stress model to capture the stress or strain distribution within the cell. According to immunofluorescence images and the adhesion protein assay, FAs of unstretched cells were distributed peripherally around the cells (Fig. A2), and the FAK-tyr397 level in stretched cells, which represented the level of FA assembly, decreased to $70 \%$ in the untreated group and $40 \%$ in the low- $\mathrm{Ca}^{2+}$ group. A zero-displacement boundary condition was imposed on all of the elements peripheral to the cell body to mimic FA sites in the unstretched cell. After stretching for $3 \mathrm{~s}, 30 \%$ or $60 \%$ of the zero-displacement elements were randomly selected to be switched to free boundary conditions to mimic the disassembly of FA sites of untreated and low $\mathrm{Ca}^{2+}$-treated cells, respectively.

The simulated cell with $30 \%$ of FA sites disassembled experienced a slight contraction after stretch and a high level distributed von Mises stress in the majority of the cell (Fig. 4B and C). Conversely, when $60 \%$ of FA sites disassembled, the simulated cell showed a significant contraction/shrinkage after the stretch. Moreover, the stress field within the cell was concentrated in the immediate vicinity of the FA sites, leaving the bulk area of the cell with low stress (Fig. $4 \mathrm{~A}$ and $\mathrm{C}$ ).

Taken together, our experimental results and finite element simulation showed that tonic stretch-induced transient FA disassembly attenuates the mechanical force distributions within the cell, which has a beneficial effect on the survival of alveolar epithelial cells upon stress failure.

$\mathbf{A}$

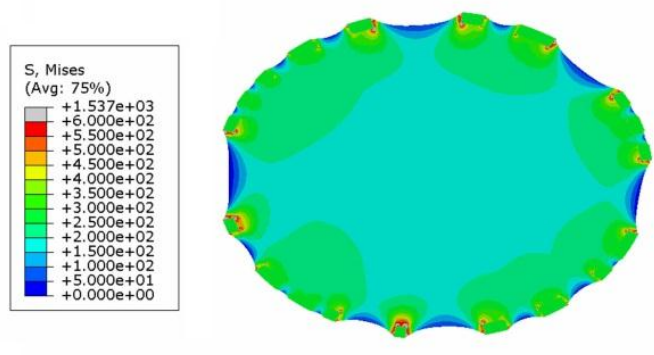

$60 \%$ FAs detachment

B
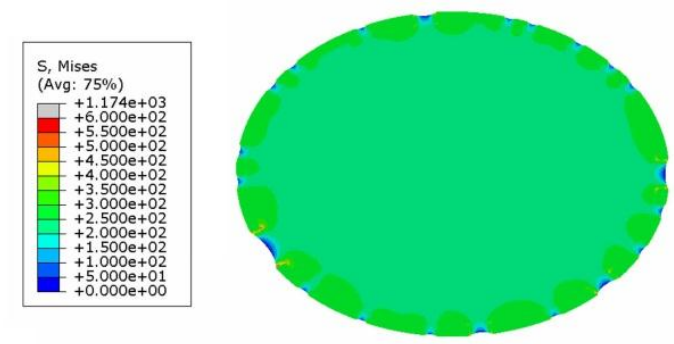

$30 \%$ FAs detachment

C

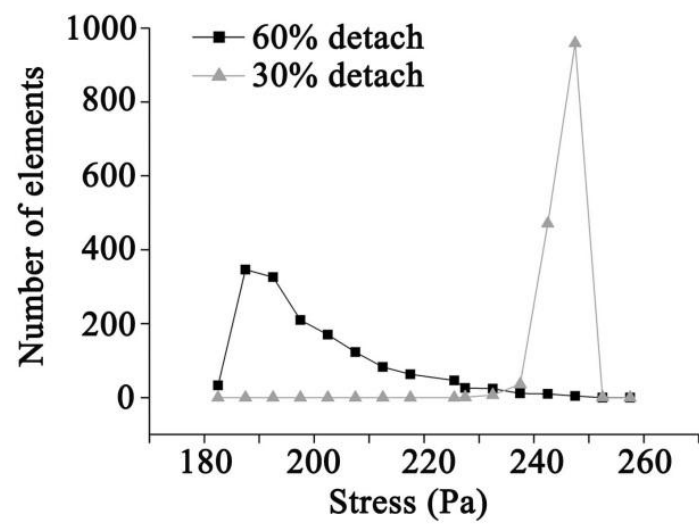

Figure 4. A continuum, viscoelastic, finite element simulation estimated the stress distribution within the cell. The cell model was applied to $37 \% \triangle \mathrm{SA}$ deformation at the beginning of the stretch, and then experienced a contraction process due to a random disassembly of the adhesion boundary. (A) The stress field within the cell with 60\% FA site disassembly was concentrated in the immediate vicinity of the FA sites, leaving the bulk area of the cell with a low stress level. (B) For the cell with $30 \%$ FA site disassembly, a high level of distributed stress was seen throughout the majority area of the cell. (C) The average stress in the cell body showed that a disassembly of $60 \%$ of FA sites might protect the bulk area of the cell from high stress. 


\section{Discussion}

Mechanical ventilation-induced excessive stretch of alveoli has been recognized to be an injurious factor to the lung. Tonic stretch, an excessive static stretch put on AECs, frequently happens in recruitment maneuver [27], high-frequency oscillatory ventilation [28], or during mechanical ventilation in ARDS patients [29], and usually causes severe AEC injury. To counteract VILI, the majority of the research has focused on either changing ventilation parameters or developing therapies that minimize the magnitude of the injurious mechanical forces [30-32]. However, an alternative approach, altering the physical characteristics of the AECs themselves to make them less susceptible to injurious mechanical forces, has not been well studied. Recently, independent reports have demonstrated that AECs can survive mechanical stimuli by insertion of lipids into the cell membrane or by increasing the fluidity of the actin cytoskeleton, suggesting new possibilities to counteract VILI. However, the mechanisms that allow AECs to survive excessive strain remain largely unknown.

In the present study, for the first time we established that ATII cells could survive tonic stretch-induced necrosis by partial detachment of FAs based on several lines of evidence as follows.

a) Using in situ SEM visualization, we observed that ATII cells underwent a contraction-reexpansion process with a large number of filaments scattered around the cell body. These filaments were actin bundles attached to the substrate, as demonstrated by immunofluorescent staining and blocking actin filament assembly. Immunoblotting of FAK phosphorylation and immunofluorescence of vinculin-staining FAs further confirmed transient FAs disassembly and sequential reassembly that occurred during the remodeling of tonic stretch-induced cells. Tonic stretch induced cell contraction, characterized by tent like structure, was due to the partial detachment of FAs. b) Enhancing integrin-ECM protein binding affinity by $\mathrm{Mn}^{2+}$ resulted in increased percentage of cell death with stretch time and significantly decreased FA disassembly. c) Low $\mathrm{Ca}^{2+}$ medium treatment, which could attenuate cell-ECM adhesion and decrease contact area of cells at acute stretch time, resulted in a low cell death rate. d) The inhibition experiment with different concentrations of an integrin neutralizing antibody showed that the extent of FA detachment is closely associated with cell survival upon tonic stretch. Thus, an adequate extent of FA disassembly, but not the detachment of the whole cell, is crucial to cell survival under tonic stretch. e) A simulated model showed that the variation of focal adhesion alters the forces transmitting throughout the cell, which may be a mechanism to allow the cell to escape stretch-induced stress failure. Taken together, our results support a crucial role for FAs in cell survival after tonic stretch.

To our knowledge, no direct experimental measurements of mechanical stress distribution throughout the cell during stretch are currently available. In the present study, a viscoelastic finite element model was presented. This model, although simplified, helps predict stress and strain distribution within the cell and shed a light on our understanding of the mechanism of cell survival after tonic stretch. According to the model, the stretched cells with $30 \%$ of FA sites disassembled, which mimics the cells in normal medium after tonic stretch, underwent a high level of membrane strain or expansion, and this result is consistent with the observation under SEM [7]. Membrane strain or expansion may induce stress failure, and cells can survive by recruiting additional phospholipids via lipid trafficking. Adequate FA detachment could protect the cells from membrane over-expansion by concentrating the stress to the local area around the FAs, which are strengthened by adjoining stress fibers. Taken together, these data suggest that both partial FA detachment and lipid trafficking contribute to the survival of the cells under tonic stretch. However, further establishment of a live experimental cell model is important to address the mechanical nature of the cytoskeleton as anisotropic fibers in a viscoelastic material.

It is reported that ventilator-induced cell plasma membrane wounding resealed after the removal of injurious stress[33]. Our experiments have demonstrated that partial FA disassembly can protect cells from tonic stretch-induced cell death, which is a way of stress unloading. It is interesting to know whether survivors of cell detachment experience plasma membrane wound resealing and further exploration of the mechanism underlying cell repair is crucial for developing novel strategies against VILI.

In summary, here we describe a novel protective response to tonic stretch in AECs by transient FA detachment, which attenuates mechanical force distribution within the cell and decreases the susceptibility to stress failure. Our results also suggest that the dynamics of FA disassembly could significantly affect AEC survival after stress failure, and disassembly of FAs may be a promising approach against VILI.

\section{Acknowledgements}

Funding was provided by the National Basic Research Program (973 Project) of China (No. 2009CB522108), the National Natural Science Foun- 
dation of China (No. 30971311 and No. 81000029) and Beijing Nova program (No. 2010B012).

\section{Conflict of Interests}

The authors have declared that no conflict of interest exists.

\section{References}

1. Ware LB, Matthay MA. The acute respiratory distress syndrome. N Engl J Med. 2000; 342:1334-1349.

2. Dreyfuss D, Saumon G. Ventilator-induced lung injury: lessons from experimental studies. Am J Respir Crit Care Med. 1998; 157:294-323.

3. Vlahakis NE, Hubmayr RD. Cellular stress failure in ventilator-injured lungs. Am J Respir Crit Care Med. 2005; 171:1328-1342.

4. Tschumperlin DJ, Margulies SS. Equibiaxial deformation-induced injury of alveolar epithelial cells in vitro. Am J Physiol. 1998; 275:L1173-1183.

5. Vlahakis NE, Schroeder MA, Pagano RE, Hubmayr RD. Role of deformation-induced lipid trafficking in the prevention of plasma membrane stress failure. Am J Respir Crit Care Med. 2002; 166:1282-1289.

6. Fisher JL, Levitan I, Margulies SS. Plasma membrane surface increases with tonic stretch of alveolar epithelial cells. Am J Respir Cell Mol Biol. 2004; 31:200-208.

7. Ren $Y$, Zhan $Q, H u$ Q, Sun B, Yang C, Wang C. Static stretch induces active morphological remodeling and functional impairment of alveolar epithelial cells. Respiration. 2009; 78:301-311.

8. DiPaolo BC, Lenormand G, Fredberg JJ, Margulies SS. Stretch magnitude and frequency-dependent actin cytoskeleton remodeling in alveolar epithelia. American Journal of Physiology-Cell Physiology. 2010; 299:C345-C353.

9. Yalcin HC, Hallow KM, Wang J, Wei MT, Ou-Yang HD, Ghadiali SN. Influence of cytoskeletal structure and mechanics on epithelial cell injury during cyclic airway reopening. American Journal of Physiology- Lung Cellular and Molecular Physiology. 2009; 297:L881-L891.

10. Vogel V, Sheetz M. Local force and geometry sensing regulate cell functions. Nature Reviews Molecular Cell Biology. 2006; 7:265-275.

11. Friedland JC, Lee MH, Boettiger D. Mechanically activated integrin switch controls \{alpha\} 5 \{beta\} 1 function. Science's STKE. 2009; 323:642.

12. Dobbs LG. Isolation and culture of alveolar type II cells. Am J Physiol. 1990; 258:L134-147.

13. Hammerschmidt S, Kuhn H, Grasenack T, Gessner C, Wirtz H. Apoptosis and necrosis induced by cyclic mechanical stretching in alveolar type II cells. Am J Respir Cell Mol Biol. 2004; 30:396-402.

14. Zhou J, Zhao L, Qin L, Wang J, Jia Y, Yao H et al. Epimorphin Regulates Bile Duct Formation via Effects on Mitosis Orientation in Rat Liver Epithelial Stem-Like Cells. PloS one. 2010;5:19-28.

15. Liu L, Wang M, Fisher AB, Zimmerman UJ. Involvement of annexin II in exocytosis of lamellar bodies from alveolar epithelial type II cells. Am J Physiol. 1996; 270:L668-676.

16. Tschumperlin DJ, Margulies SS. Alveolar epithelial surface area-volume relationship in isolated rat lungs. J Appl Physiol. 1999; 86:2026-2033.

17. Tschumperlin DJ, Oswari J, Margulies AS. Deformation-induced injury of alveolar epithelial cells. Effect of frequency, duration, and amplitude. Am J Respir Crit Care Med. 2000; 162:357-362.
18. Schober M, Raghavan S, Nikolova M, Polak L, Pasolli HA, Beggs $\mathrm{HE}$ et al. Focal adhesion kinase modulates tension signaling to control actin and focal adhesion dynamics. The Journal of cell biology. 2007; 176:667-680.

19. Karcher H, Lammerding J, Huang H, Lee RT, Kamm RD, Kaazempur-Mofrad MR. A three-dimensional viscoelastic model for cell deformation with experimental verification. Biophysical journal. 2003; 85:3336-3349.

20. Mack PJ, Kaazempur-Mofrad MR, Karcher H, Lee RT, Kamm RD. Force-induced focal adhesion translocation: effects of force amplitude and frequency. American Journal of Physiology- Cell Physiology. 2004; 287:C954-C962.

21. Dailey HL, Ricles LM, Yalcin HC, Ghadiali SN. Image-based finite element modeling of alveolar epithelial cell injury during airway reopening. J Appl Physiol. 2009; 106:221-232.

22. Hammerschmidt S, Kuhn H, Gessner C, Seyfarth HJ, Wirtz H. Stretch-induced alveolar type II cell apoptosis: role of endogenous bradykinin and PI3K-Akt signaling. Am J Respir Cell Mol Biol. 2007; 37:699-705.

23. Cox BD, Natarajan M, Stettner MR, Gladson CL. New concepts regarding focal adhesion kinase promotion of cell migration and proliferation. Journal of cellular biochemistry. 2006; 99:35-52.

24. Desai LP, Sinclair SE, Chapman KE, Hassid A, Waters CM. High tidal volume mechanical ventilation with hyperoxia alters alveolar type II cell adhesion. Am J Physiol Lung Cell Mol Physiol. 2007; 293:L769-778.

25. Bazzoni G, Ma L, Blue ML, Hemler ME. Divalent cations and ligands induce conformational changes that are highly divergent among $\beta 1$ integrins. Journal of Biological Chemistry. 1998; 273:6670-6678.

26. Hu F, Strittmatter SM. The N-terminal domain of Nogo-A inhibits cell adhesion and axonal outgrowth by an integrin-specific mechanism. Journal of Neuroscience. 2008; 28:1262-1269.

27. Halter JM, Steinberg JM, Schiller HJ, DaSilva M, Gatto LA, Landas $S$ et al. Positive end-expiratory pressure after a recruitment maneuver prevents both alveolar collapse and recruitment/derecruitment. Am J Respir Crit Care Med. 2003; 167:1620-1626.

28. Chan KP, Stewart TE. Clinical use of high-frequency oscillatory ventilation in adult patients with acute respiratory distress syndrome. Crit Care Med. 2005; 33:S170-174.

29. Terragni PP, Rosboch G, Tealdi A, Corno E, Menaldo E, Davini $\mathrm{O}$ et al. Tidal hyperinflation during low tidal volume ventilation in acute respiratory distress syndrome. Am J Respir Crit Care Med. 2007; 175:160-166.

30. Terragni PP, Rosboch GL, Lisi A, Viale AG, Ranieri VM. How respiratory system mechanics may help in minimising ventilator-induced lung injury in ARDS patients. European Respiratory Journal. 2003; 22:15s-21s.

31. De Campos T. Ventilation with lower tidal volumes as compared with traditional tidal volumes for acute lung injury and the acute respiratory distress syndrome. The Acute Respiratory Distress Syndrome Network. N Engl J Med. 2000; 342:1301-1308.

32. Brower RG, Lanken PN, MacIntyre N, Matthay MA, Morris A, Ancukiewicz $M$ et al. Higher versus lower positive end-expiratory pressures in patients with the acute respiratory distress syndrome. The New England journal of medicine. 2004; 351:327-336.

33. Gajic O, Lee J, Doerr CH, Berrios JC, Myers JL, Hubmayr RD. Ventilator-induced cell wounding and repair in the intact lung. Am J Respir Crit Care Med. 2003; 167:1057-1063. 


\section{Appendix. Detail of Numerical Model}

Finite element analysis was performed using the commercial software ABAQUS (6.9). Details about the simulations, such as the viscoelastic model, mechanical properties and material properties, were given here.

\section{Mechanical and material properties}

Living cells exhibit viscoelastic behavior. Specifically, adherent cells can be represented by a three element standard linear solid model (Fig. S1) that is analogous to a spring and fluid-like viscoelastic Maxwell model because of its solid-like character. The constitutive relation of the SLS model takes the form

$$
\sigma+\frac{\eta}{E_{2}} \dot{\sigma}=E_{1} \varepsilon+\frac{E_{1}+E_{2}}{E_{2}} \eta \dot{\varepsilon}
$$

where $E_{1}$ and $E_{2}$ are two elastic constants, and $\eta$ is a viscous constant that represents two springs and one dashpot as shown in Fig. S1. With a given strain, $\varepsilon(t)=$ $\varepsilon_{0} H(t)$, we obtain the stress relaxation function as follows:

$$
\sigma(t)=E_{1} \varepsilon_{0}+E_{2} \varepsilon_{0} e^{-\frac{t E_{2}}{\eta}}=\varepsilon_{0}\left(E_{1}+E_{2} e^{-\frac{t E_{2}}{\eta}}\right)
$$

Where $H(t)$ represents the Heaviside function. The relaxation modulus of the SLS model is then given by

$$
G(t)=E_{1}+E_{2} e^{-\frac{t E_{2}}{\eta}}
$$

according to the previous published parameters, and values for the elastic modulus were utilized to model the cell. Since the elastic modulus of AECs has been measured as 15-1800 $\mathrm{Pa}$ [21], we assume that the parameters of the three elements are $15 \mathrm{~Pa}, 100 \mathrm{~Pa}$, and $1500 \mathrm{~Pa}$ s, respectively.

All material properties were summarized in Table 1.

Table I. Characteristic parameters used in the simulation

\begin{tabular}{ll}
\hline Parameter & Model \\
\hline$E_{0}$ & $600 \mathrm{~Pa}$ \\
$E_{1}$ & $15 \mathrm{~Pa}$ \\
$E_{2}$ & $100 \mathrm{~Pa}$ \\
$\eta$ & $1500 \mathrm{~Pa} \cdot \mathrm{s}$ \\
\hline
\end{tabular}

The stress field in the cell was analyzed by an invariant of the stress tensor, Von Mises stress, which is defined as $\sigma=\left\{\frac{1}{2}\left[\left(\sigma_{x x}-\sigma_{y y}\right)^{2}+\left(\sigma_{x x}-\sigma_{y y}\right)^{2}+\left(\sigma_{y y}-\sigma_{z z}\right)^{2}+6\left(\sigma_{x y}^{2}+\sigma_{x z}^{2}+\sigma_{y z}^{2}\right)\right]\right\}^{1 / 2}$

Where $\sigma_{i j}$ is the ij-component of Cauchy's stress tensor.

Model geometry

According to the parameters measured by confocal microscopy, the alveolar epithelial cell was simplified to be an elliptical cylinder with a $40 \mu \mathrm{m}$ long axis radius, $30 \mu \mathrm{m}$ minor axis radius, and $1.5 \mu \mathrm{m}$ height [21]. Because the height was less than the other two axes, the computational model used was a plane stress model.

\section{Boundary conditions and applied load}

As cells were applied a $37 \% \Delta$ SA biaxial strain in the experiments, a displacement load was adopted in the simulations with a free stress boundary condition on the surface of the cell. According to immunofluorescence images and the adhesion protein assay, the FAs of unstretched cells were distributed peripherally around the cells (Fig. S2), and the FAK-tyr397 level of stretched cells, which represents the level of FA disassembly, decreased to $70 \%$ in the untreated group and $40 \%$ in the low $\mathrm{Ca}^{2+}$ group. According to these phenomena, we imposed a zero-displacement boundary condition on all of the elements peripheral to the cell body to mimic FA sites in unstretched cells. After stretching for $3 \mathrm{~s}, 30 \%$ or $60 \%$ of the zero-displacement elements were selected randomly to be switched to free boundary conditions to mimic the disassembly of FA sites in untreated and low Ca2+-treated cells, respectively. Then, the stretch process was applied to the simulated cells for $3 \mathrm{~s}$, and the value of the deformation was sustained for $90 \mathrm{~s}$.

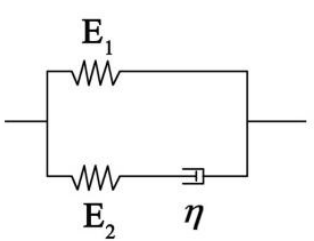

Fig. SI. The homogeneous standard linear solid (SLS) model. The whole cell is modeled as a homogeneous viscoelastic SLS. 

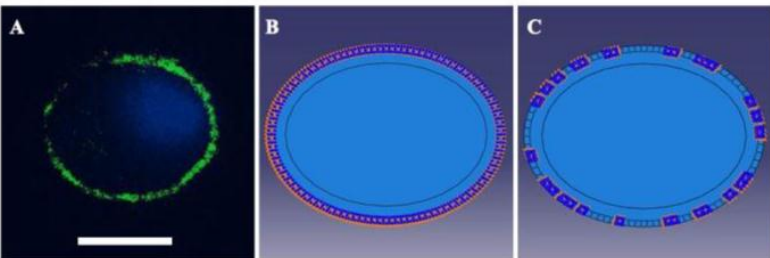

Fig. S2. (A) Immunofluorescence image showing vinculin stained FAs (green) in a peripheral distribution around the cell. DAPI was used to visualize nuclei (blue). Bar $=20 \mu \mathrm{m}$. (B) and (C). Boundary conditions used in the simulations. According to the image (A), a zero-displacement boundary condition was imposed on all of the elements peripheral to the cell body in unstretched cells (B). After stretching for 3 $\mathrm{s}, 60 \%$ of the zero-displacement elements were selected randomly to be switched to free boundary conditions $(C)$ to mimic the disassembly of FA sites in low $\mathrm{Ca}^{2+}$-treated cells.

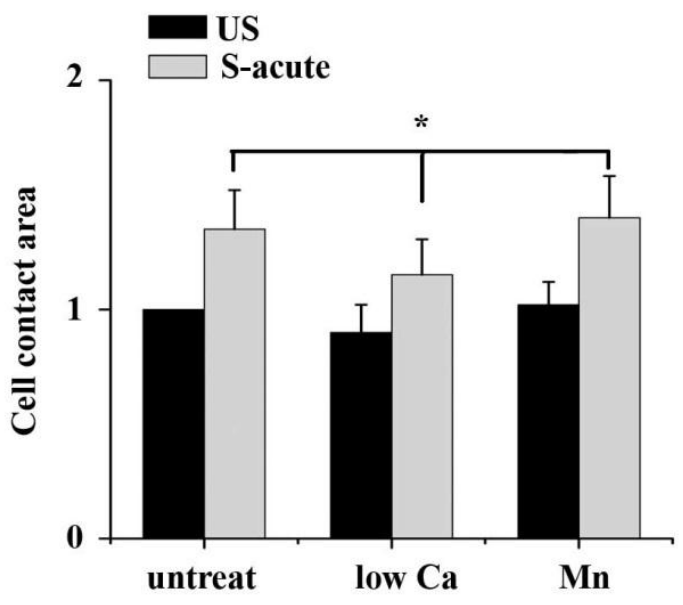

Fig. S3. By manipulating cell adhesion the ATIl cells contact area change at acute time (I $\mathrm{min})$ of tonic stretch was assessed. Cell contact area was determined by tracing the cell boundaries based on the TRITC-phalloidin staining for individual cells, conversion to a binary image, inversion of the binary image, and quantified by Images] software. Data indicated that contact area of low Ca treated cells at acute stretch time decreased compared with untreated or $\mathrm{Mn}$ treated cells $(P<0.05)$. Values were means \pm SD $(n>60)$. 